\title{
DOENÇA DE CROHN
}

\section{ISOLADA DO APÊNDICE CECAL COMO CAUSA DE ENTERORRAGIA}

\author{
Sizenando Ernesto de LIMA Jr. ${ }^{1}$, Manlio Basílio SPERANZINI ${ }^{2}$ e Marcos Pacheco GUIRO $^{1}$
}

RESUMO - Racional - A doença de Crohn isolada do apêndice é doença de paciente jovem, relativamente rara, com quadro histopatológico semelhante ao da doença de Crohn típica. Seu quadro clínico, em geral, simula o da apendicite aguda, com manifestações protraídas e na palpação do abdome, além dos sinais de irritação peritonial, nota-se com frequiência, um tumor palpável. Objetivo - Apresentar um caso de doença de Crohn isolada do apêndice que se manifestou por enterorragia e cuja origem foi identificada pela colonoscopia. Paciente - Masculino, com 16 anos de idade, sem outras manifestações digestivas apresentou dois episódios de enterorragia com intervalo de cerca de 1 ano. No segundo episódio, a colonoscopia identificou o sangramento como proveniente do óstio apendicular. Resultado - Indicada a exploração cirúrgica, constatou-se que o ceco e o íleo eram nomais e que o apêndice cecal estava com sinais inflamatórios e bloqueado pelo omento, realizando-se a apendicectomia. O exame anatomopatológico revelou tratar-se de processo inflamatório crônico com infiltrado linfoplasmocitário e granulomas esparsos, sugestivo de doença de Crohn. Em acompanhamento clínico, não apresentou manifestações de doença digestiva e a colonoscopia, realizada após 2 anos, não mostrou evidências de recidiva ou propagação da doença para o íleo. Conclusão - Neste caso, como nos demais relatados na literatura, a apendicectomia é possivelmente curativa, devendo o paciente ser acompanhado, pelo menos, por 5 anos. No diagnóstico diferencial das enterorragias em pacientes jovens, a doença de Crohn isolada do apêndice também pode ser considerada.

DESCRITORES - Apêndice. Doença de Crohn. Hemorragia g astrointestinal.

\section{INTRODUÇÃO}

A doença de Crohn isolada do apêndice cecal (DCIA), também denominada apendicite granulomatosa, é doença incomum, tendo sido descritos, desde o trabalho pioneiro de MEYERDING e BELTRAM ${ }^{(7)}$ em 1953 até o momento, pouco menos de duas centenas de casos. Incide em $0,2 \%$ a $0,6 \%$ de todos os apêndices ressecados ${ }^{(8,9)}$.

A DCIA atinge jo vens, principalmente nas décadas de 20 a 30 anos, ha vendo certa predominância do sexo masculino. Tem como característica o quadro anatomopatológico semelhante ao da doença de Crohn (DC) típica. Além disso, estando localizada apenas no apêndice cecal, cura-se possivelmente pela apendicectomia e, na maioria das vezes, não progride para outras partes do trato digestivo.

Pela oportunidade que se teve de acompanhar o caso de um adolescente com esta afecção, as dif iculdades diagnósticas e as dúvidas quanto a sua real ligação com a DC estimularam a sua apresentação.

\section{RELATO DO CASO}

FCB, sexo masculino, 16 anos de idade. Em dezembro de 1999, aos 15 anos de idade, apresentou enterorragia, sem repercussão hemodinâmica, que cessou espontaneamente. Nessa ocasião, foi submetido a vários exames entre os quais colonoscopia, trânsito intestinal e cintilografia do abdome com tecnécio marcado. Com exceção da colonoscopia que identificou lesões no íleo terminal e cujo exame anatomopatológico mostrou tratar-se de hiperplasia linfóide folicular, os demais nada revelaram de anormal. Passou bem, sem queixas digestivas até dezembro de 2000, quando apresentou nova enterorragia, também sem repercussão hemodinâmica. Nessa ocasião foi examinado, não apresentando outros

Trabalho realizado no Hospital Samaritano, São Paulo, SP

Complexo Hospitalar do Mandaqui, São Paulo, SP; ${ }^{2}$ Faculdade de Medicina do ABC, Santo André, SP.

Endereço para cor respondência: Dr. Manlio Basílio Speranzini - Rua Cons. Brotero, 1505, conj. 21 - Higienópolis - 01232-011 - São Paulo, SP. 
sintomas; no exame do abdome não havia viscerome galia ou tumor palpável. Submetido a colonoscopia, o íleo foi visualizado até cerca de $15 \mathrm{~cm}$ da válvula ileocecal, apresentando mucosa normal e peristaltismo presente. O ceco tinha morfologia e distensibilidade conservadas, apresentando, no ponto do óstio apendicular, lesão irregular, friável, com áreas deprimidas e outras ele vadas, notando-se aí coágulo fixo, compatível com sangramento recente (Figura 1). Os demais segmentos colônicos se mostravam normais. O exame anatomopatológico das biopsias mostrou tratar-se de processo inflamatório crônico exsudativo intenso e ulcerado, em mucosa do tipo colônico.

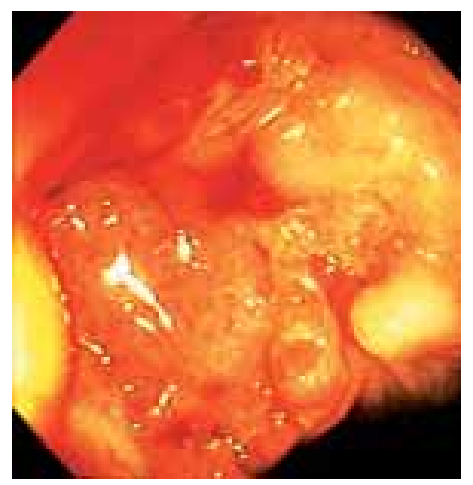

FIGURA 1 - Colonoscopia: no óstio apendicular nota-se lesão irregular, friável, de aspecto inflamatório, com áreas deprimidas, e outras com bordas elevadas, onde se nota coágulo fixo à lesão, compatível com sangramento recente (Dr. Miguel Russo Junior)

Indicada a cirurgia, realizou-se, inicialmente, uma incisão tipo McBurney. $\mathrm{O}$ apêndice cecal que se encontrava bloqueado pelo omento, foi exteriorizado com manobras digitais (Figura 2). Mostrava-se espessado e sua serosa era lisa e brilhante, tendo aderências tipo inflamatórias com o omento. Sua base era endurecida, aspecto este que se propagava circunferencialmente na parede do ceco, numa extensão de 0,5 a $1 \mathrm{~cm}$. Para possibilitar exploração mais adequada das alças intestinais, decidiu-se por realizar laparotomia mediana infra-umbilical. A exploração do intestino delgado e do restante da cavidade peritonial nada demonstrou de anormal. Após conhecer o laudo do exame histopatológico por congelação de fragmento do apêndice, decidiu-se pela sua exérese. Devido ao endurecimento da parede cecal junto ao apêndice, esta foi parcialmente (cerca de $1 \mathrm{~cm}$ ) ressecada e fechada em dois planos de sutura contínua com fio atraumático de polipropileno 5-0.

O exame histopatológico da peça cirúrgica por congelação mostrou, na parede apendicular, a existência de processo inflamatório crônico linfo-histioplasmocitário e ausência de processo neoplásico.

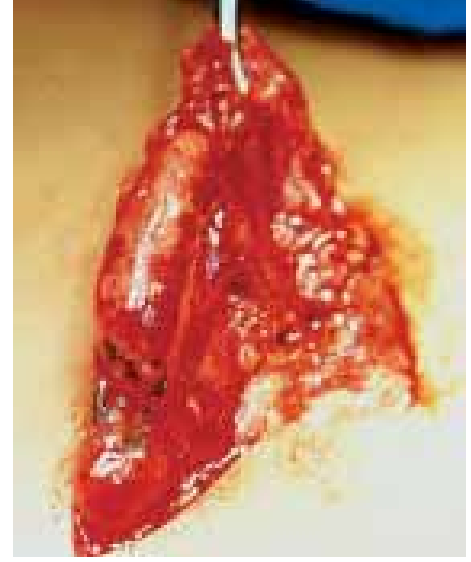

FIGURA 2 - Apêndice cecal exteriorizado por incisão tipo McBurney. O apêndice está aumentado de volume, com aderências inflamatórias do grande omento

Os cortes histológicos, após inclusão da peça em parafina constando do apêndice cecal, do omento a ele aderido e da porção do ceco ressecada, confirmaram os achados da congelação, demonstrando que o processo inflamatório era entremeado por granulomas frouxos. Na base do apêndice havia o mesmo tipo de infiltrado com granulomas, bem como a presença de ulceração da mucosa. $\mathrm{O}$ fragmento identificado como parede cecal mostrava intenso processo inflamatório crônico linfo-histioplasmocitário. Um linfonodo retirado do mesoíleo mostrava linf adenite granulomatosa. O diagnóstico final foi de DC do apêndice.

A evolução pós-operatória decorreu sem incidentes. O paciente vem sendo acompanhado em ambulatório. Em julho de 2002 (19 meses após a cirurgia), foi submetido a nova colonoscopia que mostrou íleo, cólon e reto normais. Clinicamente não apresenta queixas digestivas.

\section{DISCUSSÃO}

A maioria das casuísticas da DCIA não ultrapassa uma dezena de pacientes, sendo bastante freqüente o relato de casos (em geral um a dois) isolados ${ }^{(8,9)}$. Apesar do pequeno número de casos publicados, o interesse que desperta deriva da semelhança de seu quadro histopatológico com o da DC (daí o seu nome), mas com características peculiares - anatomopatológicas e clínico-evolutivas - que a diferenciam daquela doença. Sendo o apêndice cecal a única víscera afetada, diferentemente do que ocor re com a DC típica, recebe a denominação de doença isolada ou limitada ao apêndice.

Macroscopicamente, o apêndice se apresenta aumentado de tamanho, com acentuado espessamento da parede e com sinais inflamatórios. Estudos histológicos da DCIA e de apêndices inflamados de pacientes com DC do intestino mostraram-se comparáveis 
quanto à existência de granulomas epitelióides, infiltração focal neutrofílica de criptas com abscesso, erosões de mucosa e ulceração, fissuras, formação de fístulas, agre gados linfóides transmurais e fibrose mural. A diferenciação histopatológica entre estas duas entidades ficaria por conta da incidência de granulomas de células epitelióides que seriam muito mais numerosos na DCIA $(7,19)$ do que no apêndice da DC $(0,3)^{(5)}$. Dado o elevado número de granulomas, esta doença é também denominada de apendicite granulomatosa. A diferenciação anatomopatológica entre a apendicite granulomatosa e a apendicite da DC típica seria feita pelo granuloma. Naquela, os granulomas estariam presentes em $98 \%$ dos casos e na DC típica em apenas $60 \%{ }^{(9)}$. Contrariamente, HUANG e APPELMAN( ${ }^{(6)}$, revendo 20 casos de DCIA e comparando-os com 16 apêndices com inflamação transmural de pacientes com DC conhecida, verificaram que 11/20 pacientes com DCIA não tinham granulomas e que nos demais havia 2 a 28 granulomas por corte histológico. Os pacientes com DC conhecida nunca tinham mais do que 10,5 granulomas por corte. A ausência de granulomas assinalada por esses autores, torna difícil aceitar, para os casos de DCIA, a denominação de apendicite granulomatosa.

PRIETO-NIETO et al. ${ }^{(8)}$ ainda questionam se a DCIA seria uma forma limitada da DC ou entidade distinta denominada apendicite granulomatosa idiopática (AGI). A distinção entre a DCIA e a AGI seria histopatológica, caracterizada pela presença, nesta, de número elevado de granulomas, predominantemente na submucosa, enquanto que naquela os g ranulomas estariam mais dispersos. Além disso, na DCIA, estando presentes alterações inflamatórias agudas, pode haver ausência de granulomas, enquanto que na AGI a presença de granulomas seria indispensável. Estas diferenças levam os autores a admitir que seriam duas entidades clínicas com histopatologia distinta.

No presente caso, tanto no apêndice como no óstio apendicular, os g ranulomas eram numerosos, entremeando o processo inflamatório crônico linfo-histioplasmocitário e, na mucosa, notava-se lesão ulcerada. Aceita-se para este caso a denominação de DCIA, pois, dada a grande variabilidade conceitual entre DCIA, apendicite granulomatosa ou AGI, acredita-se que o esclarecimento desta questão somente será possível com estudos comparativos de g randes casuísticas.

O quadro clínico se caracteriza, mais freqüentemente, por dor no quadrante inferior direito do abdome, anorexia, náuseas, febre e leucocitose simulando a apendicite aguda. Chama a atenção, no entanto, o decurso protraído da sintomatologia, em geral, entre 3 e 7 dias. A presença de massa palpável na fossa ilíaca direita é outra manifestação clínica habitual ${ }^{(8,9,10)}$. No presente caso, embora à palpação não se percebesse a presença de tumor, o apêndice estava não só aumentado de volume, mas também envolto pelo grande omento (Figura 2).

O sangramento retal, como o apresentado pelo paciente desta casuística, é manifestação rara nesta doença. Encontrou-se referência a dois outros casos em que a enterorragia foi a manifestação dominante ${ }^{(3,8)}$, porém somente o presente caso teve comprovação objetiva do sangramento através da colonoscopia. É de se notar que a colonoscopia anterior, realizada após o primeiro episódio de enterorragia, não conseguiu localizar a sua origem. No caso descrito por BROWN e PETERS ${ }^{(3)}$ a provável origem do sangramento digestivo foi uma pequena lesão nodular protrusa na base do apêndice, muito semelhante à lesão que o paciente deste relato apresentava.

Dadas as manifestações pouco características da DCIA, o diagnóstico pré-operatório cor reto dificilmente é feito, sendo os casos rotulados, em geral, como apendicite aguda ou abscesso apendicular. Neste caso, o ultra-som permite demonstrar o apêndice aumentado de volume e edemaciado ou um abscesso periapendicular, não havendo sinais característicos que permitam o diagnóstico da DCIA.

Quando a manifestação é de enterorragia em paciente jovem, um dos diagnósticos mais lembrados é o do divertículo de Meckel sangrante, razão pela qual o paciente em estudo foi submetido a cintilografia com tecnécio marcado. Não é infreqüente também, especialmente durante o ato operatório, suspeitar-se de neoplasia, dado o volume aumentado da víscera ${ }^{(3,4)}$. No entanto, antes de se proceder a operações mais extensas, impõe-se como foi feito no caso em estudo, a realização do e xame histopatológico de congelação que deverá ser confimado, posteriormente, após inclusão em parafina.

O diagnóstico de DCIA exige a exclusão de várias entidades. Entre as doenças infecciosas que apresentam g ranulomas no apêndice, citam-se as provocadas pelo bacilo da tuberculose, por fungos (blastomicose, actinomicose, histoplasmose) e parasitas (esquistossomose). Estas afecções têm características anatomopatológicas que permitem, com certa facilidade, identificá-las. Já a infecção por Yersinia pseudotuberculosis é mais difícil de ser excluída, sendo necessário cultura e testes sorológicos para seu diagnóstico ${ }^{(9)}$.

A apendicectomia simples é o tratamento de escolha, apresentando mínima morbidade. Nos casos em que a base do apêndice é larga e a infiltração atinge a parede do ceco, recomenda-se a ressecção parcial do mesmo (cecectomia parcial), como se procedeu no caso presente ${ }^{(9)}$.

A diferenciação entre a DC típica e a DCIA fica por conta da evolução pós-operatória, pois, nesta última afecção, a cura pela apendicectomia isolada é quase a re gra. A formação de fístula enterocutânea após apendicectomia é raramente vista na DCIA, enquanto que ocorre entre $15 \%$ e $20 \%$ dos pacientes com DC ileocecal.

Na DCIA são citadas recidivas da DC em outras localizações do sistema digestório entre $3,5 \%$ e $6 \%$ dos $\operatorname{casos}^{(9)}$, enquanto que na DC típica a recidiva ocorre entre $34 \%$ e $58 \%$ dos $\operatorname{casos}^{(1,10)}$. Há, no entanto, inúmeros relatos de casos de DCIA seguidos por vários anos, sem que se verifique recidiva da doença ${ }^{(2,9)}$. Na casuística de 10 casos de PRIETO-NIETO et al. ${ }^{(8)}$ submetidos a apendicectomia, desenvolveu-se fístula enterocutânea em 1. Estes pacientes foram seguidos, 
em média, 14,5 anos ( 2 a 25 anos) e em nenhum deles a DC se desenvolveu em outras áreas do sistema digestório ${ }^{(8)}$.

O paciente deste relato está sendo acompanhado em ambulatório, sem queixas digestivas e sem evidências colonoscópicas da doença após 2 anos. Dada a benignidade da afecção e convencidos da eficácia do tratamento, propôs-se retornos anuais para exame clínico e a realização de colonoscopias anuais ou bianuais durante 5 anos.

Lima Jr SE, Speranzini MB, Guiro MP. Crohn's disease isolated of the appendix as a source of enteror rhagia. Arq Gastroenterol 2004;41(1):60-63.

ABSTRACT - Background - Crohn's disease confined to the appendix is relatively rare as a sole primary manifestation of the disease. Young people are more affected. The medical history and the physical examination are similar to the findings in acute appendicitis, but the manifestations are protracted. On physical examination there are signs of peritoneal irritation and an abdominal mass is palpable in the right iliac fossa. Aims - To report a case of Crohn's disease confined to the appendix and presenting with enteror rhagia. The source of the bleeding was localized by colonoscopy. Patient - A 16year old caucasian male without past history of gastrointestinal symptoms, presented with two episodes of enteror rhagia within a period of one year. In the second episode colonoscopy identified the appendicular ostium as the source of bleeding. Results - At operation the cecum and terminal ileum were normal in thickness and texture, and an inflammatory appendix adherent to the omentum was removed. Microscopically there were non-caseating granulomas, intense inf iltration of the wall with plasma cells, 1 ymphocytes and macrophages. The patient has not suffered recurrence, and a colonoscopy realized 2 years after the operation did not show signs of Crohn's disease. Conclusion - This case, like others in the literature, appendectomy is curative, but a 5-year follow-up is mandatory. When a young patient presents with enteror rhagia, this diagnosis has to be considered.

HEADINGS - Appendix. Crohn disease. Gastrointestinal hemor rhage.

\section{REFERÊNCIAS BIBLIOGRÁFICAS}

1. Ariel I, Vinograd I, Hershlag A, Olsha O, Argov S, Klausner JM, Rabau MY Freund U, Rosenmann E. Crohn's disease isolated to the appendix: truths and fallacies. Hum Pathol 1986;17:116-21.

2. Bak M, Andersen JC. Crohn's disease limited to the vermiform appendix. Acta Chir Scand 1987;153:441-6.

3. Brown WK, Peters RW. Crohn's disease of the appendix presenting as lowe intestinal hemorrhage and cecal mass. Am J Gastroenterol 1976;4:349-52.

4. Cerdán FJ, Balsa T, Torres-Melero J, García MC, Remezal M, Balibrea JL. Enfer medad de Crohn apendicular. Rev Esp Enferm Dig 1995;87:331-4.

5. Dudle y TH, Dean PJ. Idiopathic granulomatous appendicitis or Crohn's disease of the appendix re visited. Hum Pathol 1993;6:595-601.
6. Huang JC, Appelman HD. Another look at chronic appendicitis resembling Cronh's disease. Mod Pathol 1991;9:975-81.

7. Meyerding EV, Bertram HF. Nonspecific g ranulomatous inflammation (Crohn's disease) of the appendix: a case report. Surgery 1953;34:891-4.

8. Prieto-Nieto I, Perez-Robledo JP, Hardisson D, Rodriguez-Montes JA, LarrauriMartinez J, Garcia-Sancho-Martin LO. Crohn's disease limited to the appendix. Am J Surg 2001;182:531-3.

9. Richards ML, Arberger FJ. Granulomatous appendicitis: Crohn's disease, atypical Crohn's, or not Crohn's at all? J Am Coll Surg 1997;185:13-17.

10. Vanek VW, Spiros G, Awad M. Isolated Crohn's disease of the appendix. Two cases reports and a review of the literature. Arch Surg 1988;123:85-7.

Recebido em 17/4/2003 Aprovado em 15/8/2003 